\title{
Formar Psicólogos: Un Enfoque desde la Integración de Tres Concepciones
}

\section{The Formation of Psychologists: An Approach from the Integration of Three Conceptions}

\author{
Alberto Labarrere S. \\ Pontificia Universidad Católica de Chile \\ Lourdes Ilizástigui D. \\ Universidad Santo Tomás de Chile \\ Ana Tania Vargas Alfaro \\ Universidad Cardenal Raúl Silva Henríquez
}

(Recepción: Septiembre 2002 - Aceptación: Marzo 2003)

\begin{abstract}
El presente artículo aborda una de las problemáticas centrales de la formación de profesionales de la psicología; en él se hace una aproximación al proceso formativo de los psicólogos desde la óptica de tres concepciones integradas, con la finalidad de ofrecer un enfoque holístico susceptible de abarcar un rango más amplio de ámbitos formativos.

Se parte del criterio de que la contradicción entre una intencionalidad que propende a la formación de profesionales comprometidos con la realidad y el devenir educacional en los diversos países; profesionales con implicación y responsabilidad ante las instituciones formadoras, y el devenir de las profesiones de una parte y, de otra, la puesta en marcha de proyectos educacionales y formativos, en la actualidad no ofrecen la cobertura ni el espacio requerido para que los estudiantes se incorporen de manera consciente a los propósitos y actividades que tienen lugar. La idea central es que el acceso a la formación desde dimensiones formativas y explicativas de los procesos, puede propiciar una acción más coherente en la práctica cotidiana y en la teoría que la sustenta, generar dispositivos de aprendizaje y enseñanza; así como aproximarnos a criterios estimativos del desarrollo profesional más ajustados a las demandas de la formación.

En aras de tal finalidad, se integran de manera coherente una propuesta basada en los enfoques de la Unidad entre Crecimiento Institucional y Proceso Formativo; otra referida a la formación concebida como Participación Trascendente de los estudiantes y, por último la teoría de la Profesionalización Temprana y el Profesional en Formación. Palabras claves: Formación profesional, participación, profesionalización temprana.
\end{abstract}

This article addresses a main problem in the realm of the professional formation of psychologists; it is an approach to the process of becoming a psychologist started from three integrated conceptions offering an holistic view able to include a wide scope of formative processes and fields.

Authors point out the existing contradiction between the aims of forming professionals, that lye in practically all curricula, and reality. From this point of view the authors propose that, at the present time professional formation of psychologists does not offer the cover and the required space in order for students to consciously realize the intentions and activities that take place. The central idea is that currents conceptions that give access to the explanatory dimension of the processes, are neither capable of giving us a substantial and coherent theory nor to generate appropriated devices of professional leaming and training for the newest generations of psychologists. For the sake of such purpose, authors integrate, in a coherent way, a conception based on the Institutional Growth, the Participation of the students and, finally the theory of the Early Professionalization and The Professional in Formation.

Key words: Professional formation, participation, early professionalization.

\section{Introducción}

Los últimos veinte años han sido testigo de esfuerzos sistemáticos para producir transforma-

Correspondencia: Alberto Labarrere S., Facultad de Educación, Pontificia Universidad Católica de Chile. E-mail: lasar222@yahoo.com ciones profundas en prácticamente todos los sistemas educacionales. Se han movilizado recursos personales, tecnológicos, científicos y económicos con la finalidad de generar programas y proyectos formativos que superen cualitativamente a los de antaño; aparecieron modelos y aproximaciones teóricas, así como de acción práctica que, sin lugar a duda, contribuyeron a 


\section{LABARRERE, ILIZÁSTIGUI Y VARGAS}

modificar muchas de las concepciones imperantes hasta no hace mucho tiempo y, con ello, el quehacer programático y pedagógico-didáctico en la Educación Superior.

Sin embargo, hay algo que permanece inamovible: la transformación educacional continúa percibiéndose predominantemente como una conjunción de esfuerzos a realizar por las estructuras técnico administrativas y los profesores; mientras que los estudiantes no son vistos como agentes de la misma.

Es evidente la contradicción entre una intencionalidad que propende a la formación de profesionales comprometidos con la realidad y el devenir educacional en los diversos países; profesionales con implicación y responsabilidad ante las instituciones formadoras, y el devenir de las profesiones de una parte $y$, de otra, la puesta en marcha de proyectos educacionales y formativos que no ofrecen la cobertura ni el espacio requerido para que los estudiantes se incorporen de manera consciente a los propósitos y actividades transformadores que tienen lugar. Este es un dilema que debe abordarse plenamente, en busca de soluciones conducentes a una participación cada vez más amplia de los educandos.

La ausencia de una presencia participativa de los estudiantes en los procesos de transformación educacional, repercute profundamente tanto en el desarrollo, como en el destino de las transformaciones que se pretenden y, sobre todo, en la propia formación profesional, de ahí que propongamos una nueva aproximación, más coherente e integral, a la participación de los estudiantes en los mencionados procesos.

En la propuesta convergen concepciones desarrolladas por los autores a lo largo del tiempo que apuntan hacia un mismo objetivo al presentar elementos constitutivos comunes y generar implicaciones de orden práctico similares para el diseño y realización de los programas y proyectos educacionales en las universidades.

Aún cuando el alcance de la propuesta trasciende el ámbito de la formación del psicólogo y penetra en contextos de formación profesional más amplios, en esta ocasión hemos querido limitarlas al primero y compartir algunas reflexiones al respecto.

Las aproximaciones que aquí se integran son las ofrecidas por las ideas acerca de la Unidad entre Crecimiento Institucional y Proceso Formativo, desarrollada por Ilizástigui (1999; 2000;
2001); a partir de la cual se concibe el crecimiento de la institución como un proceso de "maduración" multidimensional, determinado por un conjunto amplio de factores. Desde este punto de vista, la formación que brinda la institución no aparece en calidad de resultado del crecimiento institucional, sino como uno de sus pilares.

La segunda aproximación que integramos, se refiere a la formación enfocada como Participación Trascendente de los estudiantes en los propósitos y actividades formativos diseñados por la institución, vía para promover el surgimiento del protagonismo en los educandos. Esta concepción desarrollada por Vargas (1998; 1998a; 2000; 2001) nos introduce en ámbitos proyectivos y de práctica formativa, que se conciben como inserción y construcción progresiva de espacios de participación-desarrollo.

Por último se integra la teoría de la Profesionalidad Temprana y el Profesional en Formación (Labarrere, 1996; 1997; 1997a; 1998; 1998a; 2000), la cual otorga un nuevo significado a la posición del estudiante y sus interacciones con los otros agentes del proceso formativo, al concebir un rol profesional temprano y una ampliación progresiva de sus responsabilidades y atribuciones.

Nuestra propuesta de integración para las tres aproximaciones tiene en la actualidad carácter de proyecto, su viabilidad estará determinada por ajustes ulteriores que deben producirse en el orden de la implementación práctica para obtener mayor efectividad y eficiencia; no obstante hemos querido presentarla aquí como bosquejo con la intención de colaborar al debate en marcha.

\section{Transformación educacional y participación estudiantil}

Una de las mayores dificultades y peligros a que se enfrentan los intentos de modificación educacional en la actualidad, consiste en que las acciones de transformación educacional suelen provenir fundamentalmente de las estructuras técnico administrativas y de dirección, dejando en segundo plano a los profesores y los estudiantes. Contrariamente a los propósitos, los programas de modificación en este ámbito operan de forma limitada en la conciencia y la práctica de quienes deberán ser sus "ejecutores", careciendo, por tanto, de la dinámica y estabili- 
dad necesaria para hacerlas irreversibles y sustentables.

Como alguno de nosotros señaló en una oportunidad, las instituciones educacionales hoy se debaten entre el dilema de la pasividad y el protagonismo (Labarrere, 1996a). La superación de este dilema depende de que las instituciones se incorporen de manera consciente y efectiva a la proyección transformativa y la asuman como tarea cotidiana y permanente, esencia de su transcurrir, convirtiéndose en cogestores de los intentos por modificar la realidad educacional. Para ello no basta con la simple ejecución y puesta en práctica de directivas y proyectos de cambio, reforma, perfeccionamiento, etc. surgidos en otras esferas; sino que se requiere la construcción de instancias y formas de participación desde la actividad cotidiana, en sinergia con los macroproyectos; instancias de actividad que incorporen la autorregulación y la movilización de todos los agentes de la transformación. Al efecto, tal vez resulta de mayor importancia, la modificación de la perspectiva y la mentalidad en los "niveles de base" en el sentido de concebirse como actores principales.

Asumida la participación de la institución formativa en calidad de eje de la verdadera transformación, esta no puede plantearse como una simple incorporación de los profesores y directivos técnico administrativos a nivel de las unidades docentes, sino que, en consecuencia, debe avanzarse en la apertura de espacios que promuevan y permitan la presencia participativa de todos los agentes, los estudiantes incluidos.

Sin embargo, las experiencias hoy indican que a nivel de institución, continúa relegándose a un segundo plano, cuando se tiene en consideración, la participación de los estudiantes en los procesos de transformación educacional. Parece estar claro que las unidades de formación superior actúan con una mirada desde la cual los estudiantes se conciben como agentes que deben cumplir prioritaria o únicamente la función "histórica" de aprender, prepararse para la vida; pero esta es una función que al llevar la impronta de la época, conserva la posición, los roles y las atribuciones tradicionales de los estudiante y sólo de manera muy limitada los incorpora a las tareas del perfeccionamiento educacional; desaprovechando las potencialidades que la propia institución ha ido generando en ellos durante la formación.

Como mostraremos más adelante, es posi- ble y necesario concebir que los estudiantes están llamados a desempeñar un papel nada despreciable en los intentos de reforma educacional. Este papel pueden desarrollarlo desde múltiples direcciones, y una de ellas consiste en su participación desde el propio proceso formativo y desde los momentos de su incorporación a la institución.

Un ejemplo de lo que afirmamos puede darse. Las tentativas de los centros por obtener niveles de desempeño cualitativos más altos, parecen ser percibidas como tareas que deben asumir esencialmente los agentes de dirección técnico-administrativa y los profesores; es una postura proveniente de una imagen de la acción e intervención especializada, en la cual quedan excluidos los estudiantes. La agencia de estos últimos en los movimientos en aras de la calidad, por lo común se limita a tomarlos como elemento de constatación de productos logrados; es decir utilizarlos como medio de verificar indirectamente si la enseñanza ha sido de calidad, a partir de que los conocimientos, habilidades, estrategias, vinculados a los aprendizajes programados, se muestren en situaciones e instrumentos especialmente diseñados.

Ahora bien, es factible concebir a los estudiantes desde otra perspectiva, como movilizadores de la transformación cualitativa, pero para que esto sea realizable se requiere un cambio en la visión de la institución, los roles y atribuciones de los estudiantes, los ángulos de su actividad que resultan relevantes y los espacios de participación que les son propios.

\section{La Concepción de la Unidad entre Crecimiento Institucional y el Proceso Formativo}

Elaborada por Ilizástigui (2000; 2001), la concepción parte del hecho palmario de que por lo común el crecimiento o desarrollo de las instituciones educacionales directamente encargadas de la formación, ya sea en el nivel superior u otros, se concibe exclusivamente como un producto de la acción de los factores "especializados", es decir la acción de los directivos, el personal técnico, los profesores y todos los agentes encargados de diseñar, rediseñar, administrar y llevar acabo la educación directamente en el aula.

El punto relevante que destaca esta concepción, es que los estudiantes quedan prácticamen- 
te excluidos de los propósitos y actividades transformativas, y esto tiene lugar a todo lo largo de la formación, con independencia de su nivel de desarrollo. La idea fundamental de esta aproximación radica en que el crecimiento de la institución depende de la manera en que el proceso formativo se concibe no sólo como una actividad dirigida a formar al estudiante, sino también a poner este proceso formativo en función del crecimiento institucional. Más claramente, la medida en que el desarrollo que van obteniendo los estudiantes se revierte en las fuentes que lo promueven.

Como ha expresado la autora, esta situación genera en los estudiantes una representación de sí mismos como sujetos que tienen como tarea solamente estudiar y que sólo de manera limitada se sienten comprometidos con el crecimiento de la institución en que se forman, dado que su misión en ella es sólo recibir el producto de una buena educación que los hará devenir buenos profesionales, capaces de desempeñarse en los diferentes ámbitos de la vida.

La concepción de Ilizástigui propone que los estudiantes deben concebirse como un agente activo frente a la institución y que el desarrollo de esta, y su preparación para realizar su tarea educativa, depende de la medida en que logra incorporarlos a los procesos de desarrollo; por ejemplo el perfeccionamiento en aras de una educación cualitativamente superior. De manera que se establece una relación recíproca entre los estudiantes y la institución.

Al considerar a los estudiantes como una fuerza relevante en la transformación de la institución en que se forman, la autora asume la acción recíproca como uno de los vértices del

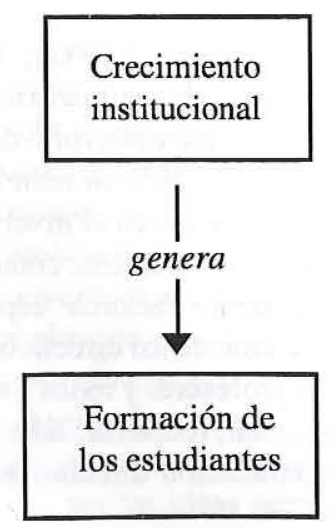

Figura 1. Concepción tradicional. desarrollo. Este recurso a la relevancia de la reciprocidad en las acciones e influencias de los agentes en los procesos formativos es fuertemente compartido por los tres autores y constituye uno de los ángulos de la comprensión de un sistema relacional más complejo (Labarrere, 1998), en correspondencia con las exigencias de la sociedad y de la educación contemporánea.

\section{La concepción de la Participación Trascendente y el Protagonismo}

La noción de Participación Trascendente ha sido desarrollada por Vargas en diferentes trabajos $(1998,1998 \mathrm{a} ; 2000 ; 2001)$ con la finalidad de argumentar e ilustrar los procesos de inserción activa de los estudiantes en los diferentes contextos (docente, familiar, laborales y comunitarios) donde transcurre su formación y participan.

La autora, sostiene que de manera general, el poder desarrollador de la enseñanza viene dado esencialmente por la posibilidad que brinda al alumno para actuar, con conciencia de la necesidad de su inserción activa en el proceso enseñanza aprendizaje y en los mencionados contextos. Tal condición de desarrollo, válida en un sentido general, se hace mucho más posible y necesaria en la enseñanza superior. En este sentido, resulta esencial que los estudiantes sean conscientes de que los procesos de aprendizaje en los que participan suponen transformación y desarrollo, y no sólo para sí mismos, sino para su grupo; e incluso, que dicha transformación no únicamente opera en el sentido de los estudiantes; sino que incluye también a los profesores. La conciencia y los significados construi-

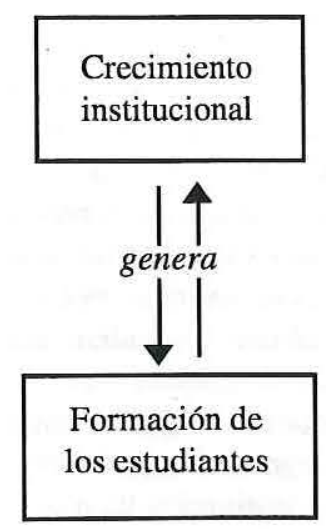

Figura 2. Concepción de integración. 


\section{FORMAR PSICÓLOGOS}

dos en torno a la participación y el desarrollo resultan, a este respecto, piedra angular de la formación.

La conciencia del rol que debe desarrollar en el aprendizaje constituye, a la vez, un producto y una condición de la ampliación de la posibilidad participativa del estudiante. Considerada en una dimensión amplia y general, la participación está en el centro de toda propuesta de desarrollo porque a su alrededor se tejen las relaciones de poder, las posibilidades de incidencia en la transformación de la calidad de vida, los marcos democráticos de acción y las posibilidades protagónicas de los sujetos.

Desde nuestro punto de vista (Vargas 2000), los factores procesales y formativos de la participación son los más relevantes a los efectos del desarrollo del estudiante universitario, pues en la medida en que el proceso participativo se haga más consciente y profundo, desde un enfoque transparente y con finalidad desarrolladora, es decir, se patentice su condición formativa mediante el crecimiento individual y grupal, los aspectos concernientes al acceso y la distribución del poder, así como la posibilidad de decidir se van convirtiendo en logros paulatinos.

En opinión de Vargas la participación trascendente tiene entre otros los siguientes atributos:

- No se instaura de manera espontánea, requieren de la formación

- Es una acción reestructuradora, orientada a la transformación y desarrollo de los sujetos

- Adquiere significación en los procesos interactivos

- Requiere de la introspección, el autoconocimiento, y la autorregulación para tomar conciencia de las transformaciones subjetivas grupales e individuales

- Establece paulatinamente la relación simetría-asimetría funcional entre la acción interventiva y la de participación.

En correspondencia con su noción de Participación Trascendente, la autora expresa que sólo a través de ella es factible aproximarse al protagonismo de los estudiantes como dimensión participativa. Para ella el alcance de una posición protagónica no puede ser visto como un hecho mecánico y directo, inducido desde fuera (desde la enseñanza y la educación, por ejemplo); sino que más bien se corresponde con un legítimo proceso constructivo. Por tal razón, el "suministro" de conocimientos o la formación de estrategias y habilidades, sean estas generales o específicas, que supuestamente permitirían el desempeño competente en diferentes campos de la actividad humana, resulta insuficiente para garantizar la intervención activa y consciente de los alumnos en y respecto al proceso formativo.

La concepción de protagonismo así expuesta, tiene como base la adquisición progresiva de responsabilidad por parte de los estudiantes, para lo cual se requiere la apertura de espacios de actividad donde sean objetos específicos de atención los siguientes aspectos que trazan el perfil del protagonismo de los estudiantes: 1) la identidad y el sentido de pertenencia; 2) la responsabilidad ante la acción en el contexto cultural; 3) la intencionalidad en la intervención; 4) la orientación al desarrollo como finalidad, y 5) la autorregulación en la actuación consciente.

Cada una de las componentes dimensionales del protagonismo constituye, a la vez, eje de la acción educativa y generatriz de criterios para estimar el desarrollo de los estudiantes desde su inserción participativa en los diferentes contextos educacionales. Para nosotros, los aspectos señalados resultan cardinales porque representan el punto de tránsito hacia la implicación, no sólo intelectual, sino también afectivo motivacional y valorativa, de los alumnos en los procesos formativos y de desarrollo que puedan gestarse.

Como puede apreciarse, entre la concepción de la participación trascendente y aquella de la unidad entre desarrollo institucional y el proceso formativo existen fuertes líneas de intersección. La que podemos trazar hasta el momento, (Fig.3) es que la comprensión de los estudiantes como agentes de transformación y crecimiento institucional pueden y deben realizarse desde una postura que considere los procesos de participación de estos y que permita basarse en criterios útiles y efectivos para estimar su desarrollo; de manera tal que este requerimiento bien puede ser cubierto desde la noción de participación trascendente. 


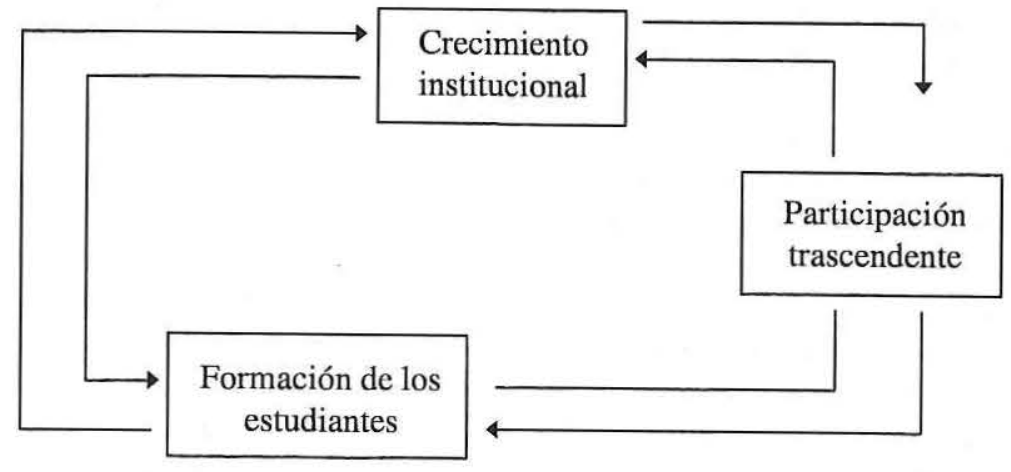

Figura 3: Interrelaciones entrre el crecimiento institucional la formación de los estudiantes y la participación trascendente.

\section{La teoría de la Profesionalidad Temprana (PT) y del Profesional en Formación}

La teoría de la profesionalidad temprana surge como una alternativa a la concepción imperante y que sobre todo de un modo implícito permea el proceso formativo de profesionales, según la cual el estudiante deviene profesional una vez que ha egresado del centro formativo, ha realizado los ejercicios formales establecidos y ostenta el título correspondiente. Tal punto de vista ha sido caracterizado como profesionalidad tardía o lejana y, como ha expuesto el autor, presenta profundas connotaciones y repercusiones para el proceso formativo de los profesionales y entre ellos los psicólogos.

La esencia de esta teoría (Labarrere, 1996; 1997; 1997a; 1998; 1998a; 2000) reside en que desde el momento mismo del ingreso al centro de formación profesional, el estudiante debe ser considerado como profesional en formación. Para la PT, quien ingresa a un establecimiento de esta naturaleza no parte de un punto cero en profesionalidad, pues dicha condición no es un momento de "entrada", sino la continuación de un proceso que se ha iniciado antes y cuyos estadios iniciales se localizan fuera de la formación profesional formalmente concebida.

Reconocer que el sujeto no "puntúa cero en profesionalidad", es decir no es una tabula rasa a este respecto cuando ingresa al centro, implica asumir que ya desde su vida estudiantil anterior, y sobre todo por su experiencia en la sociedad, la persona tiene representaciones de la profesión y de las formas de pensamiento y acción que les son propias; la elección de la profesión, que prácticamente nunca se produce totalmente al azar, menos si se ha seguido algún proceso de formación vocacional, indica su presencia. Estos conocimientos y representaciones en manera alguna son irrelevantes, y no pueden ser descartados en el proceso formativo que tendría lugar.

Así, cuando el estudiante ingresa en Psicología, él ya tiene formada una representación de cómo actúan los psicólogos, de cuáles son sus responsabilidades ante la ejecución de distintas tareas, etc. Si la referida formación vocacional ha tenido lugar, los sujetos pueden tener representaciones bastante adecuadas de la profesión. El contacto con profesionales de la familia o fuera de ella también suele favorecer el desarrollo de representaciones adecuadas de la profesión, anteriores al ingreso al centro de estudios.

Por otro lado, si la profesionalidad no surge de golpe y se considera como un proceso, entonces es necesario asumir que el estudiante va deviniendo progresivamente profesional a lo largo de su formación y surge entonces la necesidad de tener en cuenta esta cualidad a los fines del proceso formativo; orientarse en y por ella y atribuir nuevos roles, responsabilidades y atribuciones al profesional en formación, acordes con esta condición en desarrollo.

Lo anterior implica trascender el estado actual, donde los criterios para evaluar el desarrollo están totalmente vacíos respecto a la profesionalidad emergente y sólo tienen en consideración conocimientos, posibilidad de aplicar instrumentos y estrategias, etc., que si bien la componen, no la resumen ni la representan totalmente. La enseñanza no sabe, y menos el pro- . 


\section{FORMAR PSICÓLOGOS}

fesional en formación, cuánto de ese profesional está ya presente en los estudiantes al pasar de un curso a otro, qué distingue su avance; 10 más que puede decir es que el estudiante obtuvo cierta calificación o fue acreedor de determinada evaluación, que resulta capaz de aplicar uno u otro método: pero nada más relevante acerca de la profesionalidad, que es -o debe ser- el núcleo orientador del proceso formativo.

Por otro lado, reconocer tempranamente en quien se forma el estado de profesional, implica, o debe implicar, una modificación en la relación básica. Las redes de interacciones que surgen en los procesos formativos como el que nos ocupa, por lo general se describen como de profesor-alumno y entre los propios alumnos; pero la Profesionalidad Temprana conduce a que las relaciones básicas sean conceptualizadas y experimentadas por los sujetos fundamentalmente como relaciones entre colegas.

Introducir la relación entre colegas, impone la necesidad de una descentración de las posiciones clásicamente asumidas tanto por el alumno, como por quienes lo forman. Asimismo la construcción de un ambiente y un sistema de valores en que las atribuciones y los márgenes de libertad del menos experimentado, para intervenir en los procesos formativos, incluidos los tradicionalmente concebidos como práctica vinculada a la profesión, se amplíen y modifiquen cualitativamente.

En el caso de quienes se forman para psicólogos, la relación entre colegas supone que ellos tienen la posibilidad y libertad para intervenir, desde su estado de profesionalidad, en situaciones de elaboración de su propio proceso formativo, de dialogar en términos de profesionalidad con quien lo está guiando, de explorarlo como posible modelo de actuación profesional y de trazar, en gran medida, su perfil de desarrollo -a partir de un conocimiento cada vez más profundo de la profesión-, valorar los procesos formativos en que está incluido y a sí mismo como persona que ha asumido la acción formativa de manera conciente.

De hecho, lo que acabamos de decir implica que desde la Profesionalidad Temprana, los procesos autoformativos y las acciones correspondientes vienen a ocupar un lugar importante, y la ganancia progresiva en responsabilidad por parte del sujeto menos experimentado se produce mediada por un contexto de acción muy favorecedor.

En calidad de principios básicos en los que debe sustentarse la acción desde la Profesionalidad Temprana se han formulado los siguientes:

- Tratamiento de la cosmovisión, la óptica y la concepción del profesional como elementos sustanciales para el desarrollo de la identidad profesional

- Construcción de un contexto, o situación social formativa donde la profesionalidad sea un valor explícito y actuante, y en el que el "estudiante" asuma la posición de profesional en formación, desempeñándose a tono con ella en el proceso formativo

- Instaurar como unidad descriptiva, ęxplicativa y funcional la relación entre colegas

- Construcción de significados para el aprendizaje, centrados en el desarrollo profesional

- Intervención o participación de los estudiantes en su propio proceso formativo desde su profesionalidad creciente

Como puede derivarse de esta breve síntesis de la teoría, ella implica, asume y promueve un nuevo grado de participación del profesional en formación, en correspondencia total con las nociones de participación trascendente y protagonismo expuestas por Vargas. De manera tal que aspectos como la implicación, la responsabilidad, el sentido de pertenencia y el autoconocimiento y la autorreflexión devienen elementos comunes que resultan complementados con aquellos en que las concepciones difieren, permitiendo así una aproximación más integral al proceso formativo.

Es obvio, a partir de la relación expresada, que algo similar ocurre respecto a las ideas de Ilizástigui. Desde este punto de vista, la teoría de la Profesionalidad Temprana aporta las nociones de profesional en formación y relación entre colegas, que perfilan la posición desde la cual el "estudiante" genera influencias transformadoras (de desarrollo) sobre la institución, así como el contexto de interacciones o relaciones en que dichas acciones resultan legitimadas y adquieren significado. (Fig.4) 


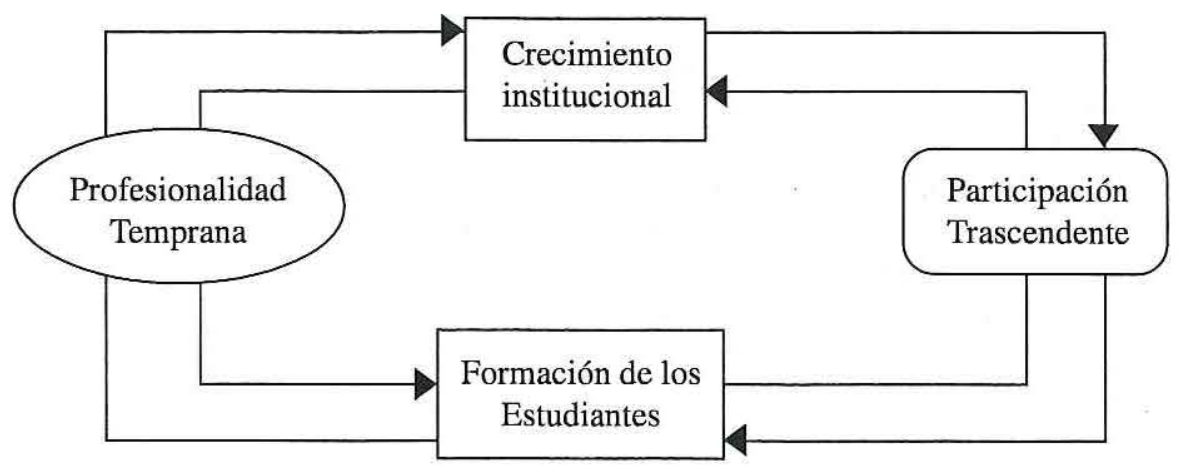

Figura 4: La integración de las tres concepciones.

\section{Qué muestran las evidencias acerca de las potencialidades de los estudiantes para participar activamente en la transformación educacional}

En primer lugar mencionamos los resultados obtenidos en investigaciones acerca de las representaciones que tienen los estudiantes de psicología sobre el proceso formativo al interior de las universidades.

En un programa de investigación acerca de las representaciones del proceso formativo que tienen los estudiantes de psicología, dirigido por Ilizástigui y Labarrere, ejecutado con la colaboración de psicólogos en formación, se ha podido comprobar que por lo común los estudiantes tienen conciencia de que el rol y las responsabilidades que se les asignan continúan siendo las de agente pasivo respecto al proceso formativo. De hecho, perciben con bastante nitidez que su lugar en el proceso docente educativo no se modifica ostensiblemente con el surgimiento de nuevas aproximaciones formativas y pedagógico-didácticas; que en buena medida su misión continúa siendo aprender, que las interacciones con sus profesores resultan excesivamente sesgadas por posiciones de autoridad y que sólo en muy baja medida les es dada la posibilidad de participar en tareas de diseño cunicular, elaboración de estrategias, etc.

Es relevante la conciencia de la necesidad del cambio de posición que tienen los estudiantes; pero en el mismo sentido debe destacarse que no todos están suficientemente seguros de si desde su posición y formación actual podrán trascender la que hoy ostentan.

La percepción de carencia participativa es clara en los señalamientos de los estudiantes; ellos se reconocen a sí mismos como pasivos y, consecuentemente experimentan la necesidad de ocupar diferente espacio; mas oscilan entre necesidad y posibilidad, estado este que ahonda la distancia existente entre los propósitos que animan las intenciones formativas del currículo y lo que ocurre en la realidad.

Los resultados expuestos muestran la complejidad del proceso formativo; puede afirmarse que en él ha generado no sólo la pasividad en los estudiantes, sino también la conciencia de la necesidad del cambio requerido; la conjugación de carencia y conciencia constituye, a nuestro juicio, una de las condiciones más importantes para transitar hacia estadios de formación cualitativamente superiores, donde los estudiantes de psicología tengan una inserción más activa y protagónica. La tarea que se plantea es, entonces, determinar las condiciones y las vías por las que dicho tránsito pude tener lugar.

En correspondencia con lo anteriormente expresado Vargas, en exploraciones de actividades de inserción en los escenarios de práctica profesional (2000), constató que cuando en la formación de los estudiantes se articulan orgánicamente actividades de reflexión acerca de los resultados de su actividad, los significados que construyen en la misma, su implicación en la formación y el desarrollo alcanzado, aumenta su capacidad y disposición para actuar de manera independiente en diferentes contextos donde transcurre la formación y, progresivamente, se va afirmando en ellos la conciencia del protagonismo y la disposición para actuar como agentes de transformación. Estamos en presencia de modificaciones en la esfera personal valorativa de los estudiantes.

Los resultados de Vargas, unidos a los obtenidos por Ilizástigui y Labarrere en otras investigaciones (no publicadas), nos informan acerca 


\section{FORMAR PSICÓLOGOS}

de las posibilidades que tienen los estudiantes para asumir responsabilidades y tareas diferentes a los que tradicionalmente se les han conferido.

El cuadro de resultados pertinentes a nuestros propósitos en esta presentación, se puede ampliar con los obtenidos por Labarrere (1998, 1999, 2000) y sus colaboradores (Sánchez, 1996; 1998). Trabajando desde el enfoque de la Profesionalidad Temprana con grupos de psicólogos en formación en una universidad mexicana, la autora puso de manifiesto que cuando, desde los años iniciales de la carrera, los estudiantes de psicología son considerados como profesionales en formación por los profesores y por ellos mismos, estableciéndose un clima de reflexión y colaboración en el diseño de las situaciones educativas, las estrategias a emplear, los dispositivos e instrumentos relevantes, entonces los primeros son capaces de asumir tareas como la del diseño de su espacio de práctica profesional, la elaboración de instrumentos de evaluación del desarrollo profesional y, lo que resulta más importante, se muestran proclives a explorar el modelo profesional que aportan los profesores y el suyo propio, derivando consecuencias relevantes al proceso formativo y para sí mismos como profesionales en formación.

Vale la pena mencionar especialmente que en sus investigaciones realizadas en la Universidad Autónoma de Morelos, México, (Sánchez, 1996) comprobó que la inserción de los estudiantes de Psicología, en contextos de profesionalidad temprana, favorecía el desarrollo de relaciones recíprocas entre ellos y los profesores, generando ambientes de intercambio favorables, donde la transformación se asumía más plenamente como responsabilidad y actividad conjunta. En esos contextos los profesores, de manera progresiva daban cabida a reflexiones de los estudiantes encaminadas a perfeccionar el currículo y los diseños instruccionales, utilizando como mediador los modelos profesionales circulantes en el contexto formativo, los cuales eran identificados, fijados y tomados como objeto de análisis y valoración sistemáticos; de manera tal que la orientación y exploración de los modelos se convertía en centro de los procesos de reflexión y diseño de acciones transformativa por parte de los estudiantes de la carrera.

Trabajando con una muestra de estudiantes de varias universidades chilenas, que en el año 2001 participaron en seminarios de tesis y talle- res de formación, y empleando una metodología destinada a explorar la Competencia y Amplitud Reflexiva de los psicólogos en formación (Labarrere, 1998), Ilizátigui y Labarrere (no publicado), se han obtenido resultados que evidencian cómo las reflexiones de los psicólogos en formación se "desplaza" de manera coherente y muchas veces sistemática por tres dominios de reflexión: 1) ellos mismos como sujetos en formación, 2) la institución como macrocontexto formativo y 3) los contenidos psicológicos generales y específicos de la disciplina. Hasta donde nos ha sido posible interpretarlos, los datos obtenidos parecen poner de manifiesto que a lo largo de la formación del psicólogo, se van conformando competencias para la reflexionar desde la profesión y acerca de ella, que no son suficientemente tenidas en consideración por el proceso formativo y permanecen relegadas a un segundo plano, a la sombra ; con lo cual se desaprovechan potencialidades que pueden emplearse en función de la propia formación y en aras de fomentar orientaciones de participación trascendente, en los términos de Vargas.

\section{En el camino de la integración y la acción consecuente}

A partir las tres concepciones aquí presentadas como integrables en un modelo coherente, capaz de ofrecer cobertura teórica y viabilidad práctica a una formación cualitativamente superior de los estudiantes de Psicología, presentaremos en síntesis nuestra propuesta.

El bosquejo realizado nos permite sintetizar de la siguiente manera idea central. Insertarse en un proceso formativo de psicólogos implica no sólo transcurrir en él sino modificar activa y conscientemente dicho proceso, reconstruirlo y transformarlo constantemente de manera útil y productiva: participar y transformar. Con esta lógica la formación deviene transformación activa de la institución poniéndose al servicio de la misma; una lógica similar podría aplicarse a otros contextos donde transcurren el aprendizaje y la educación del psicólogo.

En tal sentido, asumir la construcción de espacios de profesionalidad temprana llevaría a la institución formadora a generar una nueva mentalidad penetrada de una representación valórica e instrumental del proceso formativo, donde primaría la conciencia de que el estudiante puede y debe aportar más de lo que hoy se le consi- 


\section{LABARRERE, ILIZÁSTIGUI Y VARGAS}

dera legítimo. Su mirada estaría puesta no sólo en los procesos de aprendizaje (rendir más y mejor en el conocimiento, penetrar en sus fronteras) sino también en las condiciones donde transcurre su formación como profesional e la psicología, con la conciencia, la responsabilidad e implicación necesarias que le permitirían constituirse en agentes no sólo de su propia transformación, sino además de la de los otros.

La adscripción y desempeños de nuevos roles y responsabilidades en el proceso, generarían la necesidad de asumir nuevos criterios de desarrollo; entre los cuales los indicadores de la participación y el protagonismo, que hemos delineado, servirían como criterios de formación, estructurados para dar cuenta del desarrollo profesional; significando entonces la superación de los que hoy se erigen en torno a la adquisición de conocimientos y las habilidades profesionales, que si bien señalan un compromiso con el aprendizaje (sobre todo el propio), no lo hacen plenamente respecto a las condiciones susceptibles de mejorar a este. Dicho en otros términos, no tienen que ver obligatoriamente con los programas, los proyectos, los diseños instruccionales, etc., a través de los cuales se expresa la institución formadora como organización, cuya misión es formar profesionalmente a los estudiantes.

El modelo presentado abre las puertas para (y requiere) que el profesional en formación participe plenamente en procesos de perfeccionamiento cualitativo de la institución (acreditaciones, autoevaluaciones y evaluaciones, procesos de autonomía y así por el estilo). No menos señalable es que el profesional en formación podría implicarse en tareas de diseño de espacios y actividades de práctica profesional que incluiría aquellas que él mismo realiza o debe realizar. Generar esferas de colaboración con sus compañeros y profesores para explorar las condiciones de la formación e introducir modificaciones importantes para sí mismo y los otros.

Lo que acabamos de decir es totalmente admisible y realizable desde la óptica que integra las concepciones que aquí hemos tratado de bosquejar. Es muy posible que cada una de ellas, por separado, muestre más consistencia que las tres en unidad. A nosotros nos ha parecido viable la integración -y en ese camino andamospues creemos hay materia fértil en los núcleos centrales de las asunciones. Sobre todo porque, hasta donde sabemos, son miradas algo distintas a las tradicionales.

\section{Referencias}

Ilizástigui, L. (1999). Reflexiones, aprendizaje e Interacción en Educación Superior. Universidad Santo Tomás, Chile.

Mizástigui, L. (2000). Aprendizaje, Evaluación y Desarrollo. Universidad Santo Tomás, Chile.

Ilizástigui, L. (2001). Atención al niño con problemas de conducta: Una propuesta basada en el desarrollo personal y de la institución, Santiago de Chile.

Labarrere, A. (1996a). Escuela y Cambio Educativo: entre la Pasividad y el Protagonismo. Congreso Internacional, Pedagogía 1996, La Habana.

Labarrere, A. (1996). Formación del Psicólogo educacional y Práctica Profesional. Revista. De Cabeza, UAEM, Cuernavaca, México.

Labarrere, A. (1997a). ¿Alumno o Profesional en Formación?: Implicaciones de un Dilema para la Formación magisterial. Colecciones CIAPRO, Venezuela, No.4, 9-25.

Labarrere, A. (1997). Aprendizaje... ¿Qué le oculta la enseñanza? Revista Siglo XXI, No. 7 México, 36-44.

Labarrere, A. (1998a) Profesionalidad Temprana: Del Mito a la Realidad. Revista De Cabeza, Cuernavaca, México No. 9, 11-15.

Labarrere, A. (1998). Profesionalidad Temprana y Formación del Maestro. Revista Siglo XXI, México, No. 11, 6-14.

Labarrere, A. (2000). Aprendizaje para el desarrollo. Revista Cubana de Psicología, No. 1.

Labarrere, A. (no publicado). Profesionalidad Temprana y Formación del Psicólogo.

Sánchez, L. (1996). La formación del Psicólogo en Contextos de Profesionalidad Temprana. Tesis de Magíster, UAEM, Cuernavaca, México.

Sánchez, L. (1998). Contribución a la Utilidad de la Noción de Profesionalidad Temprana. Revista De Cabeza, No. 9, 14-17. Cuernavaca, México.

Vargas, A. (1998). Identidad y sentido de pertenencia. Dos dimensiones de un problema. Revista Siglo XXI, No. 11, México.

Vargas, A. (1998a). Promoción sociocultural y protagonismo. Contribución al diseño y ejecución del trabajo comunitario. Tesis doctoral, La Habana, Cuba.

Vargas, A. (2000). El protagonismo: Propuesta de desarrollo desde la Dimensión Cultural. Revista Cubana de Psicología, No. 1.

Vargas, A. (2001). Contexto Sociocultural, Participación y Desarrollo: Connotaciones para la Formación de los Estudiantes. Universidad Cardenal Raúl Silva Henríquez, Santiago de Chile. 